\title{
Cartesian Impedance Control For A Variable Stiffness Robot Arm
}

\author{
Florian Petit and Alin Albu-Schäffer \\ Institute of Robotics and Mechatronics, German Aerospace Center (DLR), Wessling, Germany \\ E-mails: \{Florian.Petit, Alin.Albu-Schaeffer\}@dlr.de
}

\begin{abstract}
The variable stiffness actuation (VSA) technology has been recently developed and applied in robotic arms. Mechanism robustness, high peak torque and velocity, and stiffness adjustment flexibility are key benefits of VSA joints. However, the achievable Cartesian stiffness by uncoupled VSA joints is limited. Therefore we suggest and analyze the use of an active impedance controller in combination with the passive joints to further increase the stiffness range. An algorithm to optimize the passive and active Cartesian stiffness is proposed to achieve a desired Cartesian stiffness as precise as possible. The algorithm was implemented and tested on the VSA robot DLR Hand Arm System. Experimental results and measurements of the active/passive impedance algorithm are shown.
\end{abstract}

\section{INTRODUCTION}

Cartesian impedance control is a common control framework in modern service robotics, applied to torque controlled multi-DoF robot arms and hands. While the stiffness behaviour in e.g. the DLR lightweight robot LWR3 is implemented with an active torque controller, recent developments of variable stiffness actuated robots [1], [2], [3], [4] like the DLR Hand Arm System [5] (see Fig. 1) provide passive joint compliance. The passive joint compliance is tunable online [6], so that link position and stiffness can be adjusted independently. The use of variable passive stiffness elements in robot arms has several advantages. The energy saving capability of passive springs allows to efficiently execute cyclic and highly dynamic motions, while the stiffness variability can be used to match the resonant frequencies of the robot to tasks. Furthermore, the passive springs act as low pass force filters and thereby drastically increase the robustness of the actuators against external load peaks, as they occur for example in rigid impacts of the robot with its environment.

The human arm provides biarticular muscles and thereby stiffness coupling between joints. The joint stiffness coupling in biological systems and its technical realization are still ongoing research and are not implemented in the DLR Hand Arm System. To still exploit the advantages of variable stiffness actuation, the stiffness elements in the DLR Hand Arm System are mounted separately in each joint, resulting in a diagonal joint stiffness matrix.

In an earlier work [7] we investigated what Cartesian stiffness can be achieved by a passive compliant, redundant robot arm with diagonal joint stiffness. The analysis shows that an arbitrary Cartesian stiffness matrix with three translational and three rotational stiffnesses $\left(\mathbf{K}_{C} \in \Re^{6 \times 6}\right)$ can hardly be reached by a 7 DoF arm (even using the resulting nullspace) leading to errors around 25-55\%. However, in some tasks it may be necessary to exactly attain a specified
Cartesian stiffness. One solution to this issue is analyzed in the following.

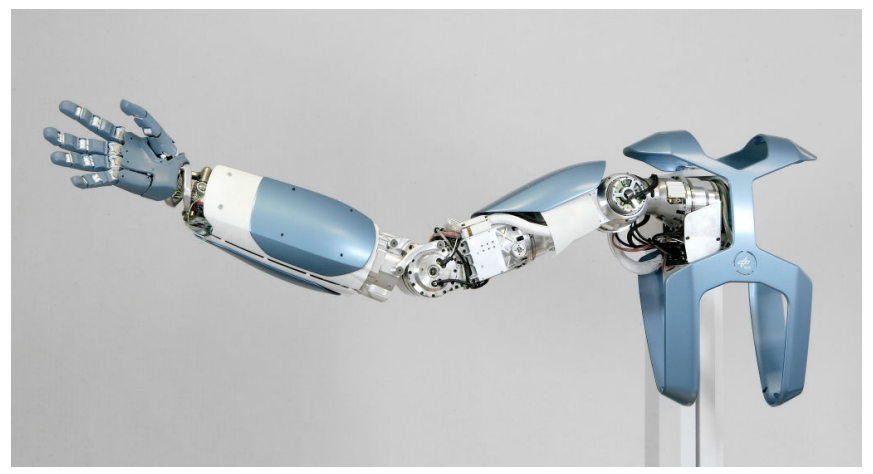

Fig. 1. The DLR Hand Arm System.

In this work we suggest to use the passive stiffness in combination with an active impedance controller [8] [9], which widely extends the achievable Cartesian stiffness range. The presented procedure involves two stages. First, the desired Cartesian stiffness is approximated using the passive joint impedance as good as possible. The passive joint stiffness is restricted by lower and upper bounds due to the technical implementation. The approximation is achieved by formulating a constrained least squares optimization problem which can be solved efficiently by an active set algorithm. Second, the residual stiffness is implemented by an active Cartesian impedance controller. The necessary positive definite active stiffness matrix is computed by a matrix nearness problem. Furthermore a compliance scaling algorithm is presented, which increases stiffness tracking and ensures bounded controller gains. The developed algorithm was implemented and evaluated on the multi-DoF variable stiffness robot DLR Hand Arm System. Finally, experimental results of the active/passive impedance controller are presented.

\section{CARTESIAN COMPLIANCE}

A general robotic task can often be intuitively specified in Cartesian coordinates. The stiffness behaviour is described by a constant stiffness matrix ${ }^{1} \mathbf{K}_{C}=-\frac{\partial \mathbf{f}}{\partial \mathbf{x}} \in \Re^{m \times m}$ as the relation between the Cartesian wrench $\mathbf{f}$ and the Cartesian displacement $\mathbf{x}$. Here, $m$ is the number of Cartesian degrees

\footnotetext{
${ }^{1}$ In the following, $\mathbf{K}_{\bullet}$ and $\mathbf{C}_{\bullet}$ is used for general stiffness/compliance matrices. Subscript ' $J$ ' and ' $C$ ' denote joint and Cartesian matrices. Subscript ' $a$ ' stands for 'active', ' $p$ ' for 'passive', ' $s$ ' for 'serial', and ' $d$ ' for 'desired' values.
} 
of freedom (DoF). The $n$ passive and adjustable joint stiffnesses provide the matrix $\mathbf{K}_{J}=-\frac{\partial \tau}{\partial \mathbf{q}} \in \Re^{n \times n}$ with the joint torques $\tau$ and the joint positions $\mathbf{q}$. The mapping from the Cartesian stiffness space to the joint stiffness space is given by $\mathcal{T}: \mathbf{K}_{J}=T\left(\mathbf{K}_{C}\right)$. This transformation can be written as

$$
\begin{aligned}
\mathbf{K}_{J} & =\frac{\partial \tau}{\partial \mathbf{q}}=\frac{\partial\left(\mathbf{J}(\mathbf{q})^{T} \mathbf{K}_{C} \Delta \mathbf{x}\right)}{\partial \mathbf{q}} \\
& =\mathbf{J}(\mathbf{q})^{T} \mathbf{K}_{C} \mathbf{J}(q)-\frac{\partial \mathbf{J}(\mathbf{q})^{T}}{\partial \mathbf{q}} \mathbf{K}_{C} \Delta \mathbf{x} .
\end{aligned}
$$

$\mathbf{J}(\mathbf{q})=\frac{\partial \mathbf{f}(\mathbf{q})}{\partial \mathbf{q}}$ is the manipulator Jacobian, where $\mathbf{f}(\mathbf{q})$ is the forward kinematics mapping. $\Delta \mathrm{x}=\mathrm{x}_{d}-\mathrm{x}$ is the infinitesimal Cartesian position error between the desired and the actual position. For further considerations in this paper the stiffness is computed at the equilibrium position. As a consequence, $\Delta \mathbf{x}=\mathbf{0}$ and (1) collapses to

$$
\mathbf{K}_{J}=\mathbf{J}(q)^{T} \mathbf{K}_{C} \mathbf{J}(\mathbf{q}) \text {. }
$$

This relation has only local correctness, as both $\mathbf{K}_{J}$ and $\mathbf{J}(\mathbf{q})$ depend upon the robot configuration.

To calculate the Cartesian stiffness from a given joint stiffness, the inverse problem of (2) has to be solved $\mathbf{K}_{C}=\mathcal{T}^{-1}\left(\mathbf{K}_{J}\right)$. This can be done considering compliance matrices, which are the inverses of the stiffness matrices $\mathbf{C}_{C}=\mathbf{K}_{C}^{-1}$ and $\mathbf{C}_{J}=\mathbf{K}_{J}^{-1}$. Please note that for inversion the matrices $\mathbf{K}_{C}$ and $\mathbf{K}_{J}$ have to be positive definite ( $\left.>0\right)$. It follows for the compliance matrices

$$
\mathbf{C}_{C}=\mathbf{J}(\mathbf{q}) \mathbf{C}_{J} \mathbf{J}(\mathbf{q})^{T}
$$

and the stiffness matrix results to be

$$
\mathbf{K}_{C}=\left(\mathbf{J}(\mathbf{q}) \mathbf{K}_{J}^{-1} \mathbf{J}(\mathbf{q})^{T}\right)^{-1} .
$$

See [7] for another computation method of $\mathbf{K}_{C}$.

To achieve an arbitrary desired Cartesian stiffness matrix $\mathbf{K}_{C}$ for a given configuration with the Jacobian $\mathbf{J}(\mathbf{q})$, in general all the elements of the joint stiffness matrix $\mathbf{K}_{J}$ must be nonzero.

\section{Problem Statement}

The passive stiffness elements in the DLR Hand Arm System adjust the stiffnesses in each joint, therefore only a diagonal joint stiffness matrix can be realized:

$$
\mathbf{K}_{J p}=\operatorname{diag}\left(\mathbf{k}_{J p}\right) \in \Re^{n \times n}
$$

$\mathbf{k}_{J p}$ is herein the joint stiffness vector. This is in contrast to the human which has coupling stiffnesses due to biarticular muscles. Furthermore, the elements of $\mathbf{k}_{J p}$ are restricted to lower and upper stiffness bounds $\mathbf{k}_{J p}^{M i n}$ and $\mathbf{k}_{J p}^{M a x}$ given by the configuration and mechanism properties.

The diagonality and boundedness of $\mathbf{K}_{J p}$ pose a substantial limitation for implementing a desired Cartesian stiffness matrix only by the passive joint stiffnesses. There are several solutions to still achieve (or get close to) an arbitrary desired Cartesian stiffness matrix $\mathbf{K}_{C}$. One approach is to exploit the redundancy of the robot arm by using the nullspace motion for stiffness optimization. This solution is analyzed in [7].

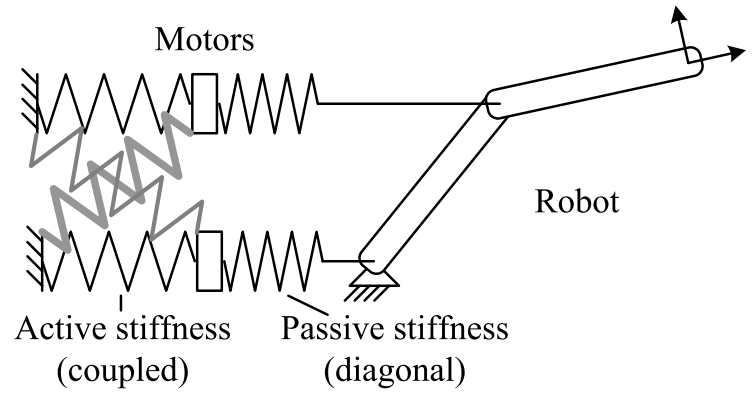

Fig. 2. The design idea to use the interconnection of an active impedance controller in series with the passive joint stiffness. As the active stiffness is less limited and may be coupled, it allows to overcome the restriction of the bounded and diagonal passive joint stiffness.

Here, we suggest and analyze the approach to use an active impedance controller in series with the passive joint impedance, in order to realize a desired Cartesian stiffness matrix $\mathbf{K}_{C d}$. As the active impedance controller is less limited (e.g. stiffnesses down to $0 \mathrm{~N} / \mathrm{m}$ can be achieved) and allows for a coupled stiffness matrix, it may overcome the limitations of the diagonal and bounded passive joint stiffnesses. This follows from writing the serial interconnection of the active and passive stiffness matrices:

$$
\mathbf{K}_{s}^{-1}=\mathbf{K}_{a}^{-1}+\mathbf{K}_{p}^{-1}
$$

This relation can be expressed by compliance matrices ${ }^{2}$

$$
\mathbf{C}_{s}=\mathbf{C}_{a}+\mathbf{C}_{p} .
$$

Obviously, even if $\mathbf{C}_{p}$ is only diagonal, the serial compliance $\mathbf{C}_{s}$ can be of arbitrary shape due to the active compliance $\mathbf{C}_{a}$. However, there are some restrictions on $\mathbf{C}_{a}$ and thereby $\mathbf{C}_{s}$, which will be discussed in Section VI-C. The design idea is sketched in Fig. 2. The desired Cartesian stiffness $\mathbf{K}_{C d}$ shall be achieved by the serial stiffness $\mathbf{K}_{C s}$. Using (6) and (4) this can be statet as an optimization problem

$$
\min _{\mathbf{K}_{J p}, \mathbf{K}_{J a}}\left\|\mathbf{K}_{C d}-\mathbf{K}_{s}\left(\mathbf{q}, \mathbf{K}_{J a}, \mathbf{K}_{J p}\right)\right\| .
$$

Besides of the stiffness matrices, the Cartesian joint stiffness depends upon the robot configuration. The problem of finding an appropriate robot configuration poses a nonlinear optimization problem (as described in [7]) and is not considered here. Therefore, the link positions are assumed to be fixed $\mathbf{q}=\mathbf{q}_{d}$ and drop out of (8).

In the following, the serial combination of the active and passive joint stiffness to achieve a desired Cartesian stiffness is analyzed and a design procedure to realize a locally valid impedance behaviour of a multi DoF VS robot arm is given.

The minimization of the norm (8) is approached in two steps. Following the conceptional idea to use mainly the passive compliance properties, first, a passive joint compliance $\mathbf{C}_{J p}$ has to be found such that the desired Cartesian

\footnotetext{
${ }^{2}$ Most of the computations involving serial interconnection of springs will be done in the compliance space in the following, as the linearity of the computations simplifies the analysis.
} 
compliance $\mathbf{C}_{C d}$ is achieved 'as good as possible' by the resulting passive Cartesian compliance $\mathbf{C}_{C p}$ (see Section IV). Afterwards in a second step, an active impedance controller using $\mathbf{C}_{C a}$ with (7) is designed such that the compliance tracking performance is increased (see Section V).

\section{PASsive Compliance Optimization}

\section{A. Problem formulation}

To optimize the passive compliance to achieve the desired Cartesian compliance ${ }^{3}$, the problem formulation from (8) is reduced to only contain the passive joint compliance vector ${ }^{4}$ :

$$
\begin{array}{ll}
\min _{\mathbf{c}_{J p}} & \left\|\mathbf{C}_{C p}\left(\mathbf{c}_{J p}\right)-\mathbf{C}_{C d}\right\| \\
\text { subject to } & \mathbf{c}_{J p}^{M i n}<\mathbf{c}_{J p}<\mathbf{c}_{J p}^{M a x}
\end{array}
$$

A similar formulation was chosen in [7], however joint stiffness limits were not regarded. Moreover, in this work we state the problem in the compliance space leading to somewhat different results.

The optimization norm was chosen to be the weighted Frobenius norm, due to computational feasibility and simplicity. The weighted Frobenius norm is defined by

$$
\|\mathbf{A}\|_{F}^{G}=\left(\sum_{i, j} g_{i j} a_{i j}^{2}\right)^{1 / 2}
$$

where $\mathbf{G}$ is a weighting matrix acting on each of the entries of the norm matrix $\mathbf{A}$.

\section{B. Algorithm}

The optimal $\mathbf{c}_{J p}$ is found by searching the extremal of (9) regarding the constraints. The first step to the solution is to transform (9) into standard least squares form by rewriting the Frobenius norm into an Euclidian norm

$$
\begin{array}{ll}
\underset{\mathbf{c}_{J p}}{\min } & \left\|\mathbf{A} \cdot \mathbf{c}_{J p}-\mathbf{b}\right\|_{2}^{G} \\
\text { subject to } & \mathbf{c}_{J p}^{M i n}<\mathbf{c}_{J p}<\mathbf{c}_{J p}^{M a x} .
\end{array}
$$

The matrix $\mathbf{A} \in \Re^{m^{2} \times n}$ contains the elements of (3) reshaped as a vector and differentiated w.r.t the joint compliance vector. The vector $\mathbf{b} \in \Re^{m^{2}}$ contains the desired compliance matrix reshaped as a vector. This inequality constrained least squares problem is solved using an active set algorithm [10]. The algorithm solves the problem as an equality constrained least squares problem where active inequality constraints are treated as equality constraints and inactive inequality constraints are omitted. The algorithm works as following:

In each iteration an equality constrained least squares problem is formulated. Herein, the inequalities from

$$
\mathbf{B}_{0} \mathbf{c}_{J p} \geq \mathbf{d}
$$

\footnotetext{
${ }^{3}$ Please note, that the norm (8) expressed by stiffness matrices can be transformed in a similar norm in the compliance space. However, weighting factors may differ.

${ }^{4}$ The passive joint compliances are written in vector form $\mathbf{c}_{J p}$ to clarify the diagonal shape of $\mathbf{C}_{J p}$.
}

where $\mathbf{B}_{0}=\left(\begin{array}{c}I \\ -I\end{array}\right)$ and $\mathbf{d}=\left(\begin{array}{c}\mathbf{c}_{J p}^{M i n} \\ \mathbf{c}_{J p}^{M a x}\end{array}\right)$, which are active are treated as equality constraints forming the working set

$$
\mathbf{B} \mathbf{c}_{J p}=\mathbf{d} \text {. }
$$

After solving the problem, two steps are performed:

1) The activeness of each equality constraint is checked by using the Karush-Kuhn-Tucker conditions, which can be done by computing the Lagrange multipliers $\boldsymbol{\lambda}$ defined by

$$
\mathbf{A}^{T}\left(\mathbf{A} \mathbf{c}_{j p}-\mathbf{b}\right)=\mathbf{B}^{T} \boldsymbol{\lambda}
$$

When all Lagrange multipliers are zero or positive, an optimal solution subject to the working set is found. In the case of a negative Lagrange multiplier, the relating equality constraint is not active and the solution is not optimal. Consequently the inactive constraint is removed from the active set and another algorithm iteration is performed.

2) The inequality constraints are checked. The constraints which are not fulfilled are treated as equality constraints and added to the working set. Then, another iteration is executed.

The algorithm terminates, if all equality constraints of the working set are active and all inequality constraints are fulfilled. This gives the passive joint compliance $\mathbf{c}_{J p}^{B e s t}$ minimizing (9) and therefore the passive Cartesian compliance $\mathbf{C}_{C p}$ nearest to the desired Cartesian compliance. Please remark, that the resulting $\mathbf{C}_{C p}$ is positive definite as all $c_{\text {Jpij }}>0$.

\section{Computation and application}

The active set algorithm is advantageous for real time computations, as the number of computation is upper bounded. At most $2^{r}$ least squares optimizations (algorithm iterations) have to be performed, where $r$ is the number of inequality constraints. Assuming a 7 DoF robot, even the worst case of 128 optimization iterations seems tractable with today's computation power.

However, in most cases the optimization is finished dramatically faster, as often only one algorithm iteration has to be performed. This is, because of in most computation steps the active compliance limits remain the same, and therefore the working set remains the same. Regarding the optimization rate of $366 \mathrm{~Hz}$ and much lower structural eigenfrequencies of the robot of $<20 \mathrm{~Hz}$ which cause the change of limits, the effectiveness of the algorithm can be understood.

If only pure passive impedance behaviour is desired, the relating passive joint stiffness $\mathbf{k}_{J p}^{B e s t}$ is computed and commanded to the robot with a high gain position controller. As mentioned in [7], the achieved stiffness performance over the workspace is quite limited. To achieve increased stiffness performance, an active impedance controller is designed to further minimize the norm (8), as shown in the next section. 


\section{Active Compliance Optimization}

The passive Cartesian compliance achieved by the optimization (9) is often not tracking the desired Cartesian compliance very well, especially when passive stiffness joint limits are considered. By interconnecting an active controller in series with the passive impedance, the tracking behaviour can be improved. This approach is analyzed in the following.

\section{A. Problem formulation}

The active Cartesian impedance controller is given by

$$
\mathbf{u}_{i m p}=-\mathbf{K}_{C}\left(\mathbf{x}-\mathbf{x}_{d}\right)-\mathbf{D}_{C} \dot{\mathbf{x}},
$$

see [11]. At this point, the question how to choose $\mathbf{D}_{C}$ is not treated. Stating the problem of finding an optimal active Cartesian stiffness again as an optimization problem derived from (8) gives

$$
\begin{array}{ll}
\min _{\mathbf{C}_{C a}} & \left\|\mathbf{C}_{C d}-\mathbf{C}_{C s}\left(\mathbf{C}_{C a}\right)\right\| \\
\text { subject to } & \mathbf{C}_{C a}>0 .
\end{array}
$$

The active compliance matrix $\mathbf{C}_{C a}$ is chosen to be positive definite, as otherwise the impedance controller (12) is not any more passive. Please remark that in theory even a negative definite $\mathbf{C}_{C a}$ may result in a positive definite serial compliance (7) and thereby the interconnection is passive, however non-ideal properties like controller time delays, measurement signal discretization and further effects are not considered and therefore may lead to instability. Furthermore, the main reason for choosing $\mathbf{C}_{C a}>0$ is that the calculations performed are only valid locally for infinitesimal deflections while in practice of course arbitrary deflections may occur leading to $\mathbf{q} \neq \mathbf{q}_{d}$ and $\mathbf{C}_{C p} \neq \mathbf{C}_{C p}^{B e s t}$. The resulting serial stiffness $\mathbf{C}_{s}$ may not be any more positive definite and may lead to instability.

With (7) and (10) the norm in (13) can be rewritten by

$$
\left\|\mathbf{C}_{C d}-\mathbf{C}_{C s}\right\|_{F}=\left\|\mathbf{X}-\mathbf{C}_{C a}\right\|_{F},
$$

where we replaced $\mathbf{C}_{C d}-\mathbf{C}_{C p}$ by $\mathbf{X}$. Therefore the minimization problem is reduced to the problem of finding a positive definite matrix $\mathbf{C}_{C a}$ closest to a desired matrix $\mathbf{X}$. This is described as a 'matrix nearness problem' in the literature.

\section{B. Optimization via a matrix nearness problem}

The matrix nearness problem of finding a positive definite matrix involving the Frobenius norm is described in [12]. The key element of the proof and the presented algorithm is to decompose the symmetric part of the goal matrix $\mathbf{X}$ into its eigenvalues and to choose only the positive values. The optimal active Cartesian compliance is computed as follows.

First, an eigenvalue transformation of the goal matrix $\mathbf{X}$ is computed:

$$
\begin{aligned}
\mathbf{X} & =\mathbf{V} \boldsymbol{\Lambda} \mathbf{V}^{T} \\
\mathbf{V}^{T} \mathbf{V} & =\mathbf{I} ; \quad \boldsymbol{\Lambda}=\operatorname{diag}(\boldsymbol{\lambda})
\end{aligned}
$$
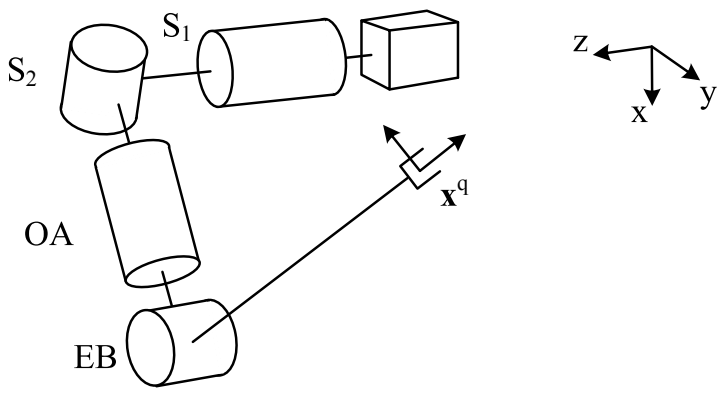

Fig. 3. A sketch of the robot configuration, which was used to conduct the experiments. Due to the position of the TCP in front of the Arm, the shoulder joint $S_{1}$ needs to produce a high torque, resulting in a high stiffness in the $x$-direction. Furthermore, the elbow with its parallel kinematics is generating high stiffnesses in the $y$-direction for this pose.

$\mathbf{V}$ is the matrix containing the eigenvectors and $\boldsymbol{\Lambda}$ is the diagonal eigenvalue matrix. Secondly, the active Cartesian compliance matrix $\mathbf{C}_{C a}$ is transformed into this eigenspace:

$$
\mathbf{C}_{Q}=\mathbf{V} \mathbf{C}_{C a} \mathbf{V}^{T}
$$

Following the proof in [12] to find the positive semi-definite matrix $\mathbf{C}_{C a}$ nearest to $\mathbf{X}$, the diagonal entries $c_{Q i i}$ of $\mathbf{C}_{Q}$ have to be chosen such that

$$
c_{Q i j}^{B e s t}= \begin{cases}\lambda_{i}, & \lambda_{i}>0 \\ 0^{+}, & \lambda_{i} \leq 0 .\end{cases}
$$

The value $0^{+}$represents a value bigger than 0 , as in contrast to the proof in [12], we need positive definiteness. The optimal active Cartesian stiffness $\mathbf{C}_{C a}$ results to be

$$
\mathbf{C}_{\mathrm{Ca}}^{\text {Best }}=\mathbf{V}^{T} \mathbf{C}_{Q}^{\text {Best }} \mathbf{V} .
$$

The optimal active compliance is transformed into stiffness space and directly used in (12).

The next section shows the use and experiments of the gained optimal active compliance $\mathbf{C}_{\mathrm{Ca}}^{\mathrm{Best}}$ in combination with the optimal passive compliance $\mathbf{C}_{C p}^{B e s t}$ from Section IV implemented on the DLR Hand Arm System.

\section{EXPERIMENTS}

\section{A. Experimental approach and procedure}

The presented algorithms were implemented and tested on the DLR Hand Arm System [5]. The first four degrees of freedom (shoulder joints one and two, the upper arm rotation joint, and the elbow joint) were used to adjust the Cartesian position $\in \Re^{3}$. Rotational stiffnesses were omitted in these first experiments. All the measurements were done with the robot in a configuration as shown in Fig. 3.

The passive joint stiffnesses in the DLR Hand Arm System are nonlinear, tunable, and dependent upon joint deflection. The joint torques are given by

$$
\boldsymbol{\tau}=\mathbf{f}(\boldsymbol{\varphi}, \boldsymbol{\sigma})
$$

where $\varphi$ is the link deflection and $\sigma$ is the stiffness preset parameter. The system allows to tune its passive joint stiffnesses via $\sigma$ in the range of $\sim 50-500 \mathrm{Nm} / \mathrm{rad}$. Passive deflection 


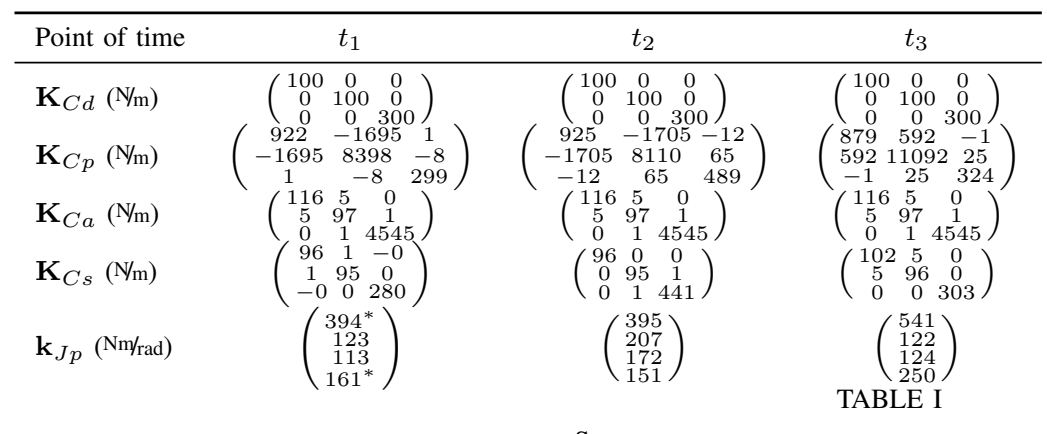

STIFFNESSES OF THE TIME INSTANTS GIVEN IN FIG. 4

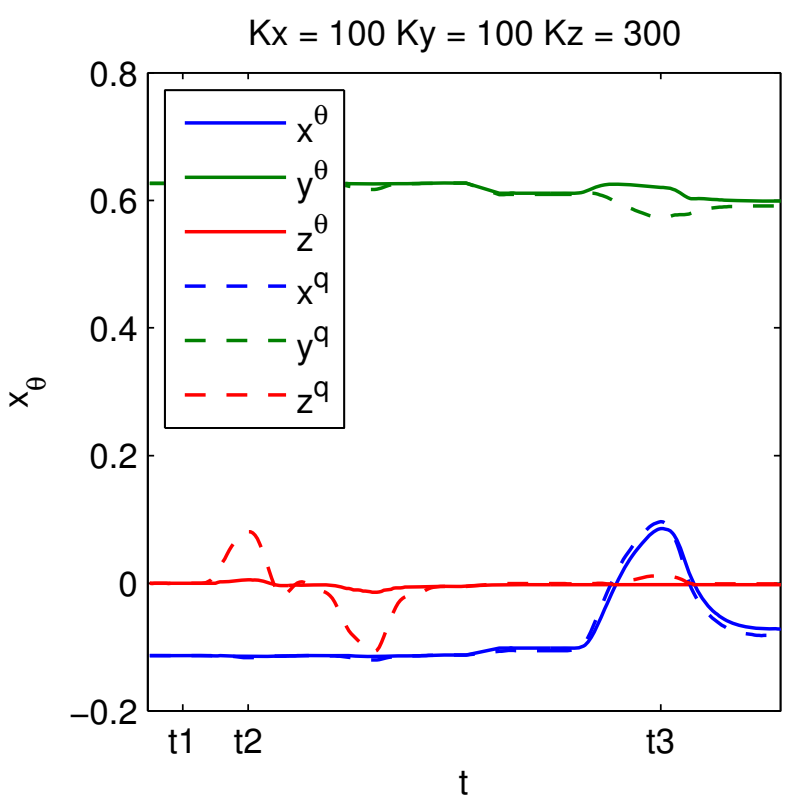

Fig. 4. Measurement plots of the TCP position $\mathbf{x}^{q}$ and motor TCP position $\mathbf{x}^{\theta}$. The TCP is deflected in the $x$ - and $z$-direction. For the case of a low passive stiffness and a high active stiffness ( $z$-direction, time point $t_{2}$ ), the passive stiffness element deflects a lot. For the case vice versa ( $x$-direction, time point $t_{3}$ ), the passive stiffness element deflects very little.

can increase the joint stiffness up to $\sim 800 \mathrm{Nm} /$ rad. The experiments were generated using the presented algorithms in the following procedure:

1) A desired Cartesian position is commanded and the desired Cartesian stiffness matrix $\mathbf{K}_{C d}$ is specified.

2) The minimum passive joint stiffness vector $\mathbf{k}_{J p}^{M i n}$ is computed. This is done by equating (15) and the robot gravity term $\mathrm{g}(\mathbf{q}, \boldsymbol{\theta})$ and numerically solving for $\varphi$. The derivative of (15)

$$
\mathbf{K}(\boldsymbol{\varphi}, \boldsymbol{\sigma})=\frac{\partial \mathbf{f}(\boldsymbol{\varphi}, \boldsymbol{\sigma})}{\partial \boldsymbol{\varphi}}
$$

where $\boldsymbol{\sigma}=\boldsymbol{\sigma}^{\mathrm{Min}}$, gives $\mathbf{K}_{J p}^{\mathrm{Min}}$.

3) The optimal passive Cartesian stiffness $\mathbf{K}_{C p}^{B e s t}$ is computed using the passive stiffness optimization from Section IV. The optimal stiffness variation parameters $\boldsymbol{\sigma}^{\text {Best }}$ to achieve the desired $\mathbf{k}_{J p}^{\text {Best }}$ are determined by a similar algorithm as presented in the last step, but

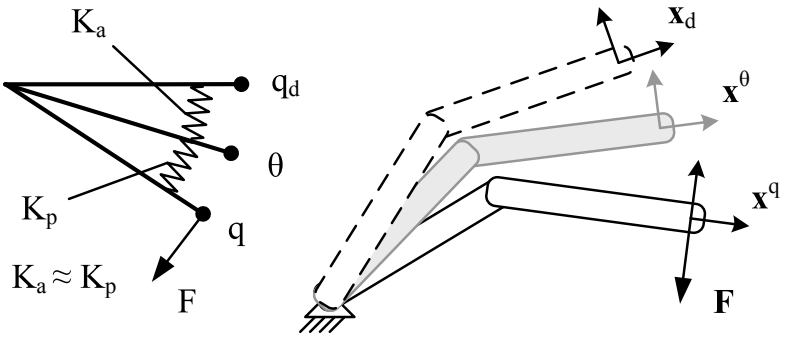

Fig. 5. On the left a one link VS joint is sketched. A force $F$ acting at the TCP deflects the link and the motor coordinate. The active stiffness $K_{a}$ is acting between the motor and the desired position while the passive stiffness $K_{p}$ is mounted between the motor and the link. On the right, the deflection behaviour can be seen for a planar robot. The motor TCP position $x^{\theta}$ is in between the desired TCP position $x_{d}$ and the TCP $x^{q}$.

solving (16) for $\sigma$.

4) The active stiffness optimization from Section V results in the optimal active Cartesian stiffness $\mathbf{K}_{\mathrm{Ca}}^{\mathrm{Best}}$.

The optimization algorithms are computed with a rate of $366 \mathrm{~Hz}$ on a real time operating system (QNX). The user chooses a desired Cartesian stiffness and triggers the parameterization of the controllers. Then the joint stiffness variation parameter $\boldsymbol{\sigma}^{\text {Best }}$ is commanded to the stiffness motors and held constant by a PD-position controller. Also the active Cartesian stiffness matrix $\mathbf{K}_{\mathrm{Ca}}^{B e s t}$ is commanded to the active Cartesian impedance controller (12) and held constant. The robot stiffness behaviour can now be analyzed by deflecting the robot from its equilibrium pose.

\section{B. Results}

Figure 4 shows measurements of the TCP position $\mathrm{x}^{q}$ and the motor based TCP position $\mathrm{x}^{\theta}$ (the motor positions instead of the link positions are used to calculate the forward kinematics, see Fig. 5). The relating stiffness values for interesting points are given in Table I. The desired Cartesian stiffness $\mathbf{K}_{C d}$ remains constant throughout the trajectory. $\mathbf{K}_{C p}$ shows the locally valid Cartesian stiffness matrix as it is generated by the passive stiffness joints. At the beginning $\left(t_{1}\right)$, the optimization from Section IV was triggered and the necessary values $\boldsymbol{\sigma}^{\text {Best }}$ were set, therefore $\mathbf{K}_{C p}=\mathbf{K}_{C p}^{\text {Best }}=$ $\mathbf{K}_{C d}$ should be achieved. However, looking at the diagonal entries, the desired value is only reached satisfactory by the $z$-coordinate $\left(K_{C p z z}=299 \mathrm{~N} / \mathrm{m}\right)$. The $x$ - and $y$-coordinates 
are not very close to the desired $\mathbf{K}_{C d}$ values. This is because of the minimal passive joint stiffness bounds prohibit to reach the low Cartesian stiffnesses: The robot configuration requires high joint torques in the first axis to support the robot and from (16) also high joint stiffnesses result (see Table I, $\mathbf{k}_{J p}$ entries marked with a ${ }^{*}$, are reaching the lower bound values). In order to compensate for the high passive Cartesian stiffness entries in $\mathbf{K}_{C p}$, the active compliance optimization computes low stiffnesses values for the $x$ - and $y$-component $\left(K_{\text {Caxx }}=116 \mathrm{~N} / \mathrm{m}, K_{\text {Cayy }}=97 \mathrm{~N} / \mathrm{m}\right)$ and high stiffness for the $z$-component. Finally, the active and passive stiffnesses sum up to a serial stiffness $\mathbf{K}_{C s}$ which is very close to the desired one.

The behaviour of the impedance Controller for deflections from the desired position can be seen in the measurements at the time instants $t_{2}$ and $t_{3}$. At $t_{2}$ the TCP is deflected in the $z$-direction. As the passive stiffness tracking in this direction is very good, most of the deflection is provided by the passive stiffness elements (large motion of $z^{q}$ in Fig. 4 ), while the high active stiffness prevents the motors from moving (almost no motion of $z^{\theta}$ ). The deflection leads to an increase of the passive joint stiffness $\left(K_{C p z z}=489 \mathrm{~N} / \mathrm{m}\right)$ because of the progressive shape of (16), which in turn results in an increase of $K_{C s z z}$, as the stiffness values are only valid locally (see also Section VII).

A similar behaviour can be seen at $t_{3}$ where a deflection in the $x$-direction is executed. As the passive joint stiffness in this direction is very high $\left(K_{C p x x}=879 \mathrm{~N} / \mathrm{m}\right)$, the TCP deflection is mainly provided by the motor deflection and thereby by the active impedance controller $\left(K_{\operatorname{Caxx}}=116\right.$ $\mathrm{N} / \mathrm{m}$ ). The apparently random change of the $y$-coordinate stems from a nullspace motion executed by the elbow joint.

Figures (6) and (7) show two more measurements of experiments of the active/passive impedance controller in action. In Fig. (6) the stiffness in the $x-y$ plane was set high, while in the $z$-direction a very low stiffness was chosen. Figure (7) shows in contrast a low stiffness in the $x-y$ plane and a high stiffness in the $z$-direction. In both setups, a commanded high stiffnesses results in a very stiff active impedance controller. A TCP deflection in these stiff directions results in a relatively large deflection of the passive stiffness elements, while the motors move only little. The low stiffness instead is mainly provided by the active impedance controller (the passive impedance is lower bounded), what appears in the plots as a large motion executed by the motors.

\section{Passive Stiffness Scaling}

The sequential procedure of first designing the passive stiffness without considering boundary values of the active compliance leads to small offsets in the serial stiffness. This is because of zero compliance is physically not possible, as the active impedance controller has upper boundaries for maximum stiffness. These offsets can be seen in the measurements of Table I for the $z$-component: $K_{C d z z}=300$ $\mathrm{N} / \mathrm{m}$ achieved closely by the passive $K_{C p z z}^{B e s t}=299 \mathrm{~N} / \mathrm{m}$. The maximum active stiffness $K_{C a z z}=4545 \mathrm{~N} / \mathrm{m}$ leads to a serial

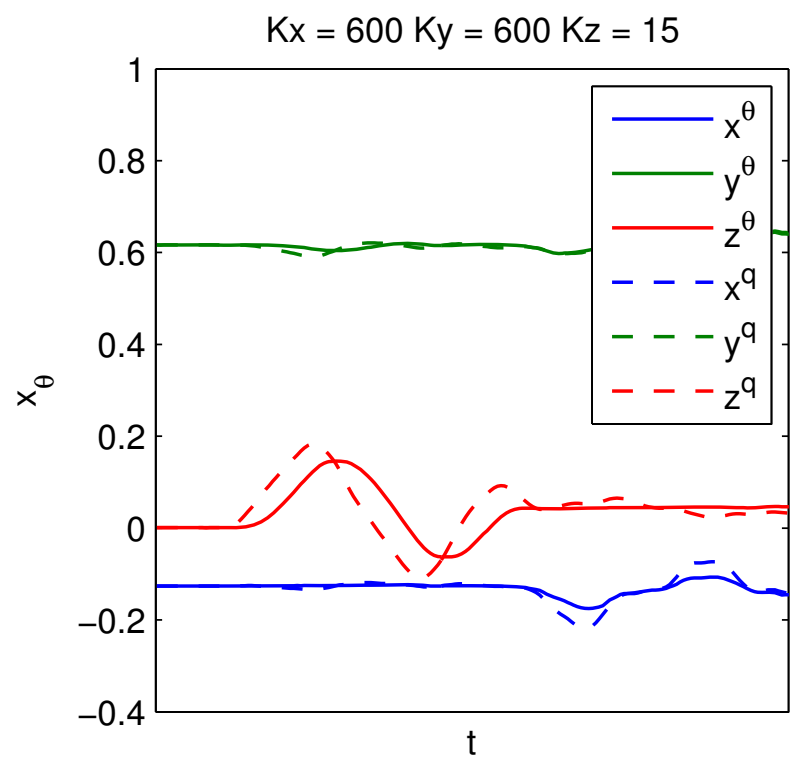

Fig. 6. Measurements of the combined impedance controller for specifying the $x-y$ plane to show high stiffness behaviour. The robot can be easily deflected in the $z$-direction, as here a very low stiffness is commanded.

stiffness a little off of the desired stiffness $K_{C s z z}=280 \mathrm{~N} / \mathrm{m}$. While this may not be relevant for real applications, two theoretical solutions are given here:

By transforming (7), where $\mathbf{C}_{s}=\mathbf{C}_{d}$, by a generalized eigenvalue decomposition into the eigenspace of $\mathbf{C}_{d}$ and $\mathbf{C}_{p}$, all matrices but $\mathbf{C}_{a}$ have diagonal shape

$$
\begin{aligned}
{[\mathbf{V}, \mathbf{\Lambda}] } & =\operatorname{eig}\left(\mathbf{C}_{p}, \mathbf{C}_{d}\right): \\
\mathbf{C}_{d Q} & =\mathbf{I}=\mathbf{V}^{T} \mathbf{C}_{d} \mathbf{V} \\
\mathbf{C}_{p Q} & =\operatorname{diag}\left(\mathbf{c}_{p Q}\right)=\mathbf{V}^{T} \mathbf{C}_{p} \mathbf{V} \\
\mathbf{C}_{a Q} & =\mathbf{V}^{T} \mathbf{C}_{a} \mathbf{V} .
\end{aligned}
$$

To find a matrix $\mathbf{C}_{a}$ which is positive definite (compare Section V), it is necessary that the diagonal elements $C_{a Q i i}>0$ and as a consequence

$$
\mathbf{C}_{p Q i i}<\mathbf{C}_{d Q i i} .
$$

However, the passive compliance optimization step from (9) does not guarantee (17) to be true. To achieve the desired property, the passive compliance matrix $\mathbf{C}_{p}^{B e s t}$ is scaled down:

$$
\mathbf{C}_{p}^{\text {Scaled }}=\frac{1}{\max \left(C_{p Q i i}\right)} \mathbf{C}_{p}
$$

Where $\max \left(C_{p Q i i}\right)$ denotes the biggest element of the diagonal matrix $\mathbf{C}_{p Q}$.

Another possibility to ensure property (17) is to consider the minimum possible compliance term already in the passive compliance optimization (9).

\section{DISCUSSION AND OUTLOOK}

The experimental results show the effectiveness of the presented algorithm. The limitations of the implementation of the Cartesian stiffness with a variable stiffness robot with 


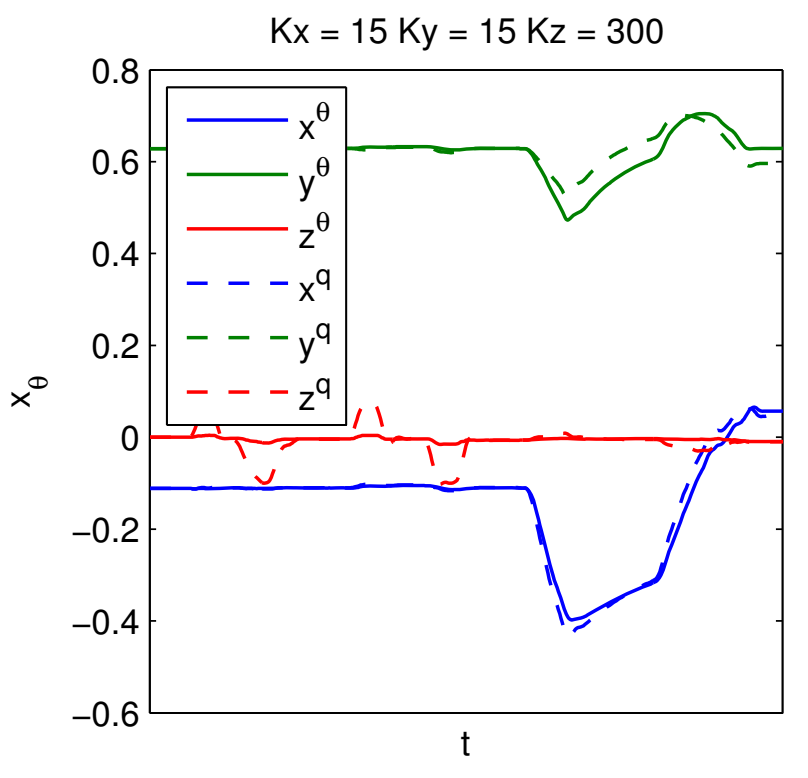

Fig. 7. Measurements of the combined impedance controller for high stiffness behaviour in the $z$-direction. The $x$ - and $y$-direction show low stiffness, which is generated mostly by the active impedance controller, as the lowest passive stiffness in the direction is relatively high (e.g. $K_{C p x x}>$ $\left.10 \cdot K_{C d x x}\right)$.

diagonal joint stiffness can be reduced by using an additional impedance controller. The combination of the active and passive impedance behaviour greatly extends the adjustable stiffness range of the robot.

Several topics will be addressed in further research. The solution to include the minimum possible compliance in the passive stiffness optimization will be further evaluated. Furthermore, the algorithm will be adopted to allow to specify a Cartesian stiffness under the influence of an external torque. Last but not least, we seek to remove the restriction of local correctness of the algorithm.

\section{ACKNOWLEDGMENTS}

The authors gratefully acknowledge the contribution of the DLR Hand Arm System team. This work has been partially funded by the European commissions Sixth Framework Programme as part of the project VIACTORS under grant No. 231554.

\section{REFERENCES}

[1] J. W. Hurst, J. Chestnutt, and A. Rizzi, "An actuator with physically variable stiffness for highly dynamic legged locomotion," in Proc. IEEE Int. Conf. on Robotics and Automation, 2004.

[2] S. A. Migliore, E. A. Brown, and S. P. DeWeerth, "Biologically inspired joint stiffness control," in Proc. of the IEEE/RSJ International Conference on Intelligent Robots and Systems, 2005, pp. 4519-4524.

[3] G. Tonietti, R. Schiavi, and A. Bicchi, "Design and control of a variable stiffness actuator for safe and fast physical human/robot interaction," in Proc. IEEE Int. Conf. on Robotics and Automation, 2005, pp. 528-533.

[4] B. Vanderborght, B. Verrelst, R. Van Ham, M. Van Damme, D. Lefeber, B. M. Y. Duran, and P. Beyl, "Exploiting natural dynamics to reduce energy consumption by controlling the compliance of soft actuators," Int. J. Rob. Res., vol. 25, no. 4, pp. 343-358, 2006.

[5] M. Grebenstein, A. Albu-Schäffer, T. Bahls, M. Chalon, O. Eiberger, W. Friedl, R. Gruber, S. Haddadin, U. Hagn, R. Haslinger, H. Höppner, S. Jörg, M. Nickl, A. Nothhelfer, F. Petit, J. Reill, N. Seitz, T. Wimböck, S. Wolf, T. Wüsthoff, and G. Hirzinger, "The DLR Hand Arm System," in Proc. IEEE Int. Conf. on Robotics and Automation, 2011.

[6] T. Wimböck, C. Ott, A. Albu-Schäfer, A. Kugi, and G. Hirzinger, "Impedance control for variable stiffness mechanisms with nonlinear joint coupling," in Proc. of the IEEE/RSJ International Conference on Intelligent Robots and Systems, 2008.

[7] A. Albu-Schäffer, M. Fischer, G. Schreiber, F. Schoeppe, and G. Hirzinger, "Soft robotics: What cartesian stiffness can we obtain with passively compliant, uncoupled joints?" in Proc. of the IEEE/RSJ Int. Conf. on Intelligent Robots and Systems, 2004.

[8] A. Albu-Schäffer, C. Ott, and G. Hirzinger, "A unified passivity based control framework for position, torque and impedance control of flexible joint robots." Int. Journal of Robotics Research, vol. 26, pp. 23-39, 2007.

[9] L. Zollo, B. Siciliano, A. D. Luca, E. Guglielmelli, and P. Dario, "Compliance control for an anthropomorphic robot with elastic joint: Theory and experiments," ASME Journal of Dynamic Systems, Measurements, and Control, vol. 127(3), pp. 321-328, 2005.

[10] A. Björck, Numerical Methods for Least Squares Problems. SIAM, 1996

[11] C. Ott, Cartesian Impedance Control of Redundant and Flexible-Joint Robots. Springer tracts in advanced robotics, 2008.

[12] N. J. Higham, "Computing a nearest symmetric positive semidefinite matrix," in Linear Algebra and its Applications, 1998. 